Moroccan J. of Pure and Appl. Anal. (MJPAA)

Volume 7(2), 2021, Pages 324-336

ISSN: Online 2351-8227 - Print 2605-6364

DOI: $10.2478 / \mathrm{mjpaa}-2021-0021$

\title{
A Partition of unity finite element method for valuation American option under Black-Scholes model
}

\author{
Zaineb El Kharrazi ${ }^{1}$, Nouh Izem ${ }^{2}$, Mustapha MaleK $^{3}$, and Sahar Saoud ${ }^{4}$
}

\begin{abstract}
In this paper, we present an intelligent combination of partition of unity (PU) and finite element (FE) methods for valuing American option pricing problems governed by the Black-Scholes (BS) model. The model is based on a partial differential equation (PDE) from which one can deduce the Black-Scholes formula, which gives a theoretical estimated value of options using current stock prices, expected dividends, the option's strike price, expected interest rates, time to expiration and expected volatility. Although the finite element method (FEM) seems to be an alternative tool for pricing options with a few applications reported in the literature, this combination called the Partition of Unity Finite Element Method (PUFEM) appears to offer many of the desired properties. The main advantage of the proposed approach is its ability to locally refine the solution by adapting an incorporated specific class of enrichment in the finite element space instead of generating a new fine mesh for the problem under study. Numerical computations are carried out to show a huge reduction in the number of degrees of freedom required to achieve a fixed accuracy which confirms that the PUFE method used is very efficient and gives better accuracy than the conventional FE method.
\end{abstract}

Mathematics Subject Classification (2020). 80M10, 65N30, 91G80.

Key words and phrases. American option, Black-Scholes, Finite element method, Enriched finite element method

Received August 30, 2020 - Accepted: January 20, 2021.

(C) The Author(s) 2021. This article is published with open access by Sidi Mohamed Ben Abdallah University.

${ }^{1}$ Engineering Sciences and Energy Management Laboratory, ENSA, Ibn Zohr University

zaineb.elkharrazi@edu.uiz.ac.ma

${ }^{2}$ Laboratory of Engineering Sciences, Faculty of Science, Ibn Zohr University

izemnouh@gmail.com

${ }^{3}$ Higher School of Education and Training, ESEF, Sultan Moulay Slimane University

malek.mustapha.masi@gmail.com (Corresponding Author)

${ }^{4}$ Technical Research Laboratory, Faculty of Applied Sciences, Ibn Zohr University

sahar.saoud@gmail.com . 


\section{Introduction}

Options are one of the most common derivatives traded in the market and it has been used for many centuries. The idea behind this derivative is to have a sort of protection or profit against the rise or fall of the price of the underlying asset.. In a financial market, an option is a contract which gives to its holder the right, and not the obligation, either to sell (put option) or to buy (call option) a quantity of a company's stock by a certain date at a given price, all fixed before in the contract. Nowadays different types of options are traded at the world exchanges, starting with simple vanilla options and continuing to high dimensional options. Therefore, there is a big interest and focus to find the correct option prices. Furthermore, option prices play a major role in risk management [9], estimation [20] and hedging [17].

In this paper we consider the problem of pricing American option, this problem has been explored in great depth in the option pricing literature even under the Black-Scholes model (Black and Scholes, 1973 [4]). Unlike the European stock option, which can be exercised at maturity only, American options can be exercised at any time up to maturity, which implies that the problem of American option is more difficult than the European option valuation, and still remains a major challenge in today's financial markets because of the associated early exercise policy that requires the option holder to make a decision prior to the contract expiration, which complicates the problem even in the single-asset case.

There are differents models for studying the behaviour of the underlying asset, such as Heston and Merton models with stochastic volatility model, yet we chose the standard BlackScholes model since it is a basic model and our aim is to reinforce the classical model using the enriched finite element approach.

In most cases, there is no analytical formula available to compute the prices of the contracts, so it becomes necessary to use numerical methods. There are various numerical methods, which are used for the valuation of financial derivatives and also in modern risk management. Generally speaking, there are two main approaches, in financial mathematics, to calculate American option prices: Partial differential equation-based methods and lattice approaches. In this paper we focus on first group which includes the finite difference (FD) method $[6,10]$, the finite volume (FV) method [21, 11], and the finite element (FE) method [3,5]. More precisely, we use the partition of unity finite element method to solve the one-dimensional Black-Scholes problem.

The success of the partition of unity finite element method is due to the fact that the partition of unity finite element method offers an easy way to include analytical information about the problem being solved in the finite element space. This method permitted the construction of finite element spaces which based on the boundary conditions behavior and then led to a robust method in the sense that the performance of the method is independent of the actual strength of the boundary conditions. It has also been proven that the enrichment significantly reduce the error achieved using an order of magnitude less degrees of freedom. At the same time comparisons to the standard finite element method show a corresponding reduction in the computational time $[15,16]$.

In financial environments with high levels of uncertainty, noise and dynamics, the BlackScholes model seems to be the basic model used for valuing option pricing. Therefore, several papers have already studied and developed this model in order to find numerical results close 
to reality with a small error [1, 22]. For a real financial configuration, the demand for a high accuracy of the resulting prices and risk measures, of online availability of prices, and the need for repeatedly performing those calculations for different input parameters as a kind of sensitivity analysis this combination makes such problem can be highly demanding computationally, see for instance $[7,2,12]$. Thus, in the present paper we compute the numerical solution of the general Black-Scholes PDE using partition of unity finite element approach which significantly improve the computational efficiency of the enriched finite elements by circumventing the need for highly reffined meshes. The comparison with the classical finite element approach shows that the proposed partition of unity method is highly accurate and robust.

The layout of this paper is as follows. In section 2 we present the model equation of BlackScholes, then Transformation of the classical model in section 3. The partition of unity finite element (PUFE) method is presented in section 4, along with enrichment functions used in the finite element solution. In section 5, we present numerical results and examples for several tests of our problems. Our PUFE approach is shown to enjoy the expected accuracy as well as the robustness. Conclusion is given in section 6 .

\section{Black-Scholes equation for Option pricing}

The Black-Scholes model has been and is still being criticised. Some of the assumptions have been disregarded. For example, dividends can be paid out discretely or continuously, and other dynamics than the Geometric Brownian motion asset dynamics have been proposed to better capture the stock behaviour. Nevertheless, in this work we adhere to the classical model, since this is not the main focus, but our focus is on the numerical method

In this section, we will assume the standard Black-Scholes model of perfect securities markets (no taxes, restrictions on short sales, transaction costs ...), where the price $S$ of the underlying asset satisfies the following stochastic differential equation,i.e Geometric Brownian motion:

$$
d S=r S_{t} d t+\sigma S_{t} d W_{t}
$$

where $d W_{t}$ is a standard Brownian motion, $r$ is the constant interest rate and $\sigma$ is the constant volatility of the underlying asset. Suppose that we have an option whose value $V(S, t)$ depends only on $S$ and $t$. To obtain the process followed by the option price at any time $t \in[0, T]$ before maturity, we must understand how $V$, the price of an option, evolves as a function of $S$ and $t$. Using Itô's lemma; equation (2.1), we can write:

$$
d V=\sigma S \frac{\partial V}{\partial S} d W+\left(r S \frac{\partial V}{\partial S}+\frac{1}{2} \sigma^{2} S^{2} \frac{\partial^{2} V}{\partial S^{2}}+\frac{\partial V}{\partial t}\right) d t
$$

We construct a portfolio consisting of one option (since we work in dimension 1) and $\Delta$ number of the underlying asset. The value of this portfolio is:

$$
\Pi=V-\Delta S
$$


We Assume that $\Delta$ is fix during the time-step, then putting (2.1), (2.2) and (2.3) together, we get that $\Pi$ follows the random walk:

$$
d \Pi=\sigma S\left(\frac{\partial V}{\partial S}-\Delta\right) d W+\left(r S \frac{\partial V}{\partial S}+\frac{1}{2} \sigma^{2} S^{2} \frac{\partial^{2} V}{\partial S^{2}}+\frac{\partial V}{\partial t}-r \Delta S\right) d t
$$

In order to eliminate the random component in (2.3), let $\Delta=\frac{\partial V}{\partial S}$ which is the rate of change of the value of our option with respect to $S$, then we obtain:

$$
d \Pi=\left(\frac{1}{2} \sigma^{2} S^{2} \frac{\partial^{2} V}{\partial S^{2}}+\frac{\partial V}{\partial t}\right) d t
$$

After choosing the quantity $\Delta$, we hold a portofolio whose value change by the amount in equation (2.5). This change is completely riskless. If we have a completely risk-free change in the portfolio value $\Pi$, then we must this equivalent equation:

$$
d \Pi=r \Pi d t .
$$

which present the no arbitrage principle.

Substituting (2.3) and (2.5) into (2.6) we find that

$$
\left(\frac{\partial V}{\partial t}+\frac{1}{2} \sigma^{2} S^{2} \frac{\partial^{2} V}{\partial S^{2}}\right) d t=r\left(V-S \frac{\partial V}{\partial S}\right) d t
$$

Dividing by $d t$ we get the Black-Scholes equation:

$$
\frac{\partial V}{\partial t}+r S \frac{\partial V}{\partial S}+\frac{1}{2} \sigma^{2} S^{2} \frac{\partial^{2} V}{\partial S^{2}}-r V=0
$$

To complete the model it is necessary to define appropriate time and boundary conditions associated with (2.2). These conditions depend on the kind of real option (call or put; European or American) being evaluated and on the numerical method to be applied. An essential boundary condition is defined in terms of the variable's value at the domain's boundary and a natural boundary condition is defined in terms of the variable's derivative value. Since we focused in the rest of this paper on American put style option, then, the terminal conditions is given by:

$$
V(S, T)=\max (K-S, 0)
$$

and the boundary conditions are:

$$
\begin{aligned}
& \lim _{S \longrightarrow 0} V(S, t)=K \\
& \lim _{S \longrightarrow \infty} V(S, t)=0 .
\end{aligned}
$$

Because of the early exercise possibility, an additional constraint:

$$
V(S, t) \geq G(S)
$$

is introduced to avoid arbitrage possibilities. Here, $G(S)=\max (K-S, 0)$ is the payoff function. 


\section{Model Transformation to Diffusion Equation}

This section is aimed at presenting intuitively the transformation of the Black-Scholes PDE to a diffusion equation and developing an analytical approximation for the American put in a simple setting.

In several papers it was shown that classical Black-Scholes model is a backward type which is difficult to solve directly. To solve the problem in (2.7) with the conditions stated in (2.7)(2.11), we need to make the model in forward type. In this regard, we introduce the following new variables:

$$
\begin{aligned}
S & =K e^{x} \\
t & =T-\frac{\tau}{\frac{\sigma^{2}}{2}} \\
v(x, \tau) & =\frac{1}{K} V(S, t)
\end{aligned}
$$

Then, from the change of variables we obtain the following derivatives:

$$
\begin{aligned}
\frac{\partial V}{\partial t} & =-\frac{\sigma^{2}}{2} K \frac{\partial v}{\partial \tau^{\prime}} \\
\frac{\partial V}{\partial S} & =\frac{K}{S} \frac{\partial v}{\partial x^{\prime}} \\
\frac{\partial^{2} V}{\partial S^{2}} & =\frac{K}{S^{2}} \frac{\partial^{2} v}{\partial x^{2}}-\frac{K}{S^{2}} \frac{\partial v}{\partial x}
\end{aligned}
$$

Substituting in equation (2.7), we get

$$
\frac{\partial v}{\partial \tau}=\frac{\partial^{2} v}{\partial x^{2}}+(\theta-1) \frac{\partial v}{\partial x}-\theta v
$$

with $\theta=\frac{2 r}{\sigma^{2}}$.

Put $\quad \lambda=\frac{1}{2}(\theta-1)$ and $\quad v=\frac{1}{2}(\theta+1)=\lambda+1$, we get

$$
\begin{aligned}
v^{2} & =\lambda^{2}+\theta \\
v(x, \tau) & =e^{-\lambda x-v^{2} \tau}\left(-v^{2}\right) u(x, \tau)+\frac{\partial u}{\partial \tau} e^{-\lambda x-v^{2} \tau} .
\end{aligned}
$$

Then,

and

$$
\frac{\partial v}{\partial x}=e^{-\lambda x-v^{2} \tau}\left(-\lambda u+\frac{\partial u}{\partial x}\right)
$$

$$
\frac{\partial^{2} v}{\partial x^{2}}=e^{-\lambda x-v^{2} \tau}\left(\lambda^{2} u-2 \lambda \frac{\partial u}{\partial x}+\frac{\partial^{2} u}{\partial x^{2}}\right) .
$$

Inserting these into equation (3.4) and dividing by $e^{-\lambda x-v^{2} \tau}$, we get: 


$$
-v^{2} u+\frac{\partial u}{\partial \tau}=\left(\lambda^{2} u-2 \lambda \frac{\partial u}{\partial x}+\left(\partial^{2} u\right) /\left(\partial x^{2}\right)\right)+(\theta-1)\left(-\lambda u+\frac{\partial u}{\partial x}\right)-\theta u
$$

Then the Black-Scholes problem reduces to the following diffusion partial differential equation:

$$
\frac{\partial u}{\partial t}-\frac{\partial^{2} u}{\partial x^{2}}=0
$$

and the initial and boundary conditions for the Put option are given by

$$
\begin{aligned}
& u(x, 0)=\left(e^{(\lambda+1) x}-e^{\lambda x}\right)^{+}, x \in \mathbb{R} \\
& u(x, t)=e^{\lambda x+\lambda^{2} t}, \quad x \rightarrow-\infty \\
& u(x, t)=0 \quad x \rightarrow+\infty
\end{aligned}
$$

Thus the Black-Scholes equation reduced to a heat diffusion equation. The benefit of this transformation is that the diffusion equation is a far simpler than the Black-Scholes. It is straightforward to find exact solutions to the diffusion equation and then convert back to financial variables.

\section{Enriched Finite Element Method}

As shown in the previous section, we are interested in solving the following problem

$$
\frac{\partial u}{\partial t}-\frac{\partial^{2} u}{\partial x^{2}}=0
$$

where $t$ is the time, $x$ is the spatial coordinate, the considered boundary conditions are given by

Initially,

$$
\begin{array}{ll}
u(x, t)=e^{\lambda x+\lambda^{2} t}, & x \rightarrow-\infty, \\
u(x, t)=0, & x \rightarrow+\infty .
\end{array}
$$

$$
u(x, 0)=u_{0}(x), \quad x \in \mathbb{R},
$$

where $u_{0}(x)$ is a known function, for the BS problem solved in this paper we have

$$
u_{0}(x)=\left(e^{(\lambda+1) x}-e^{\lambda x}\right)^{+} .
$$

To solve the problem (4.1) in a given space domain $\Omega$ with a boundary $\Gamma$, we first divide the time domain into equal intervals $\left[t_{n}, t_{n+1}\right]$ each of a duration $\Delta t=t_{n+1}-t_{n}$ where $n=0,1, \ldots$ The notation $\delta^{n}$ is used to denote the value of a generic function $\delta$ at time $t_{n}$. To integrate in time we use a semi-implicit Euler scheme in which only linear terms are integrated implicitly to avoid solution of nonlinear systems of algebraic equations. Hence, a semi-discrete form of the problem (4.1) is

$$
\frac{u^{n+1}-u^{n}}{\Delta t}-\frac{\partial^{2} u^{n+1}}{\partial x^{2}}=0,
$$


or also as

$$
u^{n+1}-\Delta t \frac{\partial^{2} u^{n+1}}{\partial x^{2}}=u^{n}
$$

For the spatial discretization, we multiply the equation in (4.4) by a weighting function $w(x)$, and then integrate over $\Omega$, we obtains

$$
\int_{\Omega} w u^{n+1} d \Omega-\Delta t \int_{\Omega} w \frac{\partial^{2} u^{n+1}}{\partial x^{2}} d \Omega=\int_{\Omega} w u^{n} d \Omega .
$$

Using the divergence theorem one may write

$$
\int_{\Omega} w \frac{\partial^{2} u^{n+1}}{\partial x^{2}} d \Omega=\int_{\Gamma} w \frac{\partial u^{n+1}}{\partial \mathbf{n}} d \Gamma-\int_{\Omega} \frac{\partial w}{\partial x} \cdot \frac{\partial u^{n+1}}{\partial x} d \Omega,
$$

then we obtain the weak formulation: Find $u^{n+1} \in H^{1}(\Omega)$ such that

$$
\int_{\Omega} w u^{n+1} d \Omega+\Delta t \int_{\Omega} \frac{\partial w}{\partial x} \frac{\partial u^{n+1}}{\partial x} d \Omega-\Delta t \int_{\Gamma} w \frac{\partial u^{n+1}}{\partial \eta} d \Gamma=\int_{\Omega} w u^{n} d \Omega,
$$

where $H^{1}(\Omega)$ is the conventional Sobolev space.

To solve the weak formation (4.7) with the finite element method, we discretize the spatial domain $\Omega$ into a set of finite elements $\mathcal{T}_{i}$ where $i$ refers to the $i$-th element. Then, the finite element solution to $u^{n}$ is formulated as

$$
u^{n}(x)=\sum_{j=1}^{M} u_{j}^{n} \phi_{j}(x),
$$

where $M$ is the number of solution mesh points and $u_{j}$ are the corresponding nodal values defined as $u_{j}=u\left(x_{j}\right) . \quad\left\{\phi_{j}\right\}_{j=1}^{M}$ are the set of global nodal basis functions characterized by $\phi_{j}\left(x_{i}\right)=\gamma_{i j}$ where $\gamma_{i j}$ denoting the Kronecker delta. Then, the finite element approximation space is defined as

$$
V^{0}=\operatorname{span}\left\{\phi_{j}, u^{n}(x)=\sum_{j=1}^{M} u_{j}^{n} \phi_{j}(x)\right\} .
$$

Solving the diffusion problems with sharp moving fronts and steep boundary layers using the finite element method require large number of degrees of freedom [18, 15]. In order to be able to capture these solution features in a coarse mesh we may enrich the finite element approximation space (4.9) using some special well-defined functions with abilities to capture the solution in the computational domain. Using the partition of unity method [14] one may enrich the solution space with enrichment functions which replicate the physical behaviour of the problem and lead to a better approximation properties compared to the conventional polynomial basis functions [18, 13]. The enrichment functions exist in many forms, examples of these enrichment functions include exponential Gaussian functions [18] and hyperbolic tangent functions [19]. In the present work, we consider time-independent global enrichment defined as

$$
G_{q}(x)=\exp \left\{-\alpha R^{q}\right\}, \quad q=1,2, \ldots, Q
$$


TABLE 1. American put option parameters

\begin{tabular}{ll}
\hline Strike & Risk-free-rate \\
\hline$K=100$ & $r=0.05$ \\
\hline
\end{tabular}

where $\alpha$ is a constant to increase / decrease the steepness of the enrichment function $G_{q}$, $R:=\left|x-x_{0}\right|$ being the distance from the control point $x_{0}$ to the point $x$ and $Q$ is the number of enrichment functions used. Then, the solution at nodes $u_{j}^{n}$ can be expanded in terms of these enrichment functions

$$
u_{j}^{n}=\sum_{q=1}^{Q} A_{j}^{q, n} G_{q}
$$

where $A_{j}^{q, n}$ is the new set of unknowns. Substituting (4.11) into (4.8) results in

$$
u^{n}(x)=\sum_{j=1}^{M} \sum_{q=1}^{Q} A_{j}^{q, n} G_{q}(x) \phi_{j}(x),
$$

where the product $G_{q}(x) \phi_{j}(x)$ form the new shap function. The approximation space can then be defined as

$$
\widetilde{V}_{h}=\operatorname{span}\left\{G_{q}(x) \phi_{j}(x), \quad u^{n}(x)=\sum_{j=1}^{M} \sum_{q=1}^{Q} A_{j}^{q, n} G_{q}(x) \phi_{j}(x)\right\} .
$$

The same enrichment functions are used at all the computational domain so that the global enrichment approach is followed.

\section{Numerical results}

In this section we aim to present the numerical results for the Black-Scholes problem using the proposed enriched finite element method. To examine the efficiency of the approach, a concrete example on American Put pricing is considered. The obtained results are compared to the results of the standard finite element method. All computations run with an Intel(R) Core i5-4210M(TM) CPU 2.60Ghz and 8GB RAM using MATLAB codes.

In order to evaluate the accuracy of the PUFEM, we solve the Black-Scholes problem in a spatial domain $\Omega=[-13,10]$ with the time to maturity $T=0.5$. The American put option parameters are summarized in Table 1 . The finite element method is used with linear finite element which is often used for solving similar problems, a high-order finite element method can also be used to solve linear and nonlinear time dependent problems, see for instance [8].

The numerical results are obtained using both FE and PUFE methods. The FEM solution is obtained on a mesh composed of 1000 nodes and is used for qualitative comparisons with the PUFEM solution. The PUFEM solution is obtained on a coarse mesh of 40 nodes and using five enrichment functions $(Q=5)$. To measure the difference between the analytical and the 
TABLE 2. Numerical solution of the Black-Scholes using FEM and PUFEM methods

\begin{tabular}{cccc}
\hline & BS-solution & FEM-solution & PUFEM-solution \\
\hline$\sigma=0.5, K=80$ & 17.9124 & 23.6769 & 23.6125 \\
$\sigma=0.5, K=100$ & 9.1308 & 12.8877 & 12.7884 \\
$\sigma=0.5, K=120$ & 4.3994 & 6.6339 & 6.5292 \\
$\sigma=0.8, K=80$ & 24.6610 & 30.1184 & 29.9943 \\
$\sigma=0.8, K=100$ & 16.7740 & 21.0196 & 20.8647 \\
$\sigma=0.8, K=120$ & 11.4410 & 14.6882 & 14.5227 \\
$\sigma=1.0, K=80$ & 29.1376 & 34.5980 & 34.4533 \\
$\sigma=1.0, K=100$ & 21.8114 & 26.3228 & 26.1491 \\
$\sigma=1.0, K=120$ & 16.4988 & 20.2116 & 20.0246 \\
\hline
\end{tabular}

TABLE 3. Relative errors obtained using the FEM and PUFEM.

\begin{tabular}{ccccccc}
\hline & \multicolumn{3}{c}{ FEM } & & \multicolumn{3}{c}{ PUFEM } \\
\hline & $\sigma=0.5$ & $\sigma=0.8$ & $\sigma=1.0$ & $\sigma=0.5$ & $\sigma=0.8$ & $\sigma=1.0$ \\
\hline$K=80$ & 0.10356 & 0.04897 & 0.03512 & 0.10126 & 0.04677 & 0.03328 \\
$K=100$ & 0.16929 & 0.06407 & 0.04278 & 0.16046 & 0.05947 & 0.03955 \\
$K=120$ & 0.25797 & 0.08055 & 0.05064 & 0.23436 & 0.07255 & 0.04567 \\
\hline
\end{tabular}

numerical solutions we consider the following relative error

$$
\epsilon=\frac{\|u-U\|_{L^{2}(\Omega)}}{\|U\|_{L^{2}(\Omega)}}
$$

where $\|\cdot\|_{L^{2}(\Omega)}$ is the $L^{2}$-norm, $u$ and $U$ are respectively, the computed and the exact solution.

In Table 2, we summarize the obtained numerical solution of the Black-Scholes problem using both methods FEM and PUFEM with different strike $K$ and volatility $\sigma$ values, the analytical solution is also presented in the same table. The solutions of this problem are computed using the nodes of $x$-domain and at the maturity time $T=0.5$. It is clear that the PUFE method can resolve Black-Scholes problem on coarse mesh with few enrichment functions. The total number of degrees of freedom in the FE method is 1000 whereas with the PUFE method this is reduced to $40 \times 5=200$. As it can be seen from table 2 , by fixing the volatility $\sigma$ and increasing the strike $K$ the stock price decrease, for example, by fixing $\sigma=0.5$ and increasing the strike from $K=80$ to $K=120$, the BS-solution decrease from 17.9124 to 
4.3994, the same conclusions can be drawn for $\sigma=0.8$ and $\sigma=1.0$. However, by fixing the strike $K$ and increasing the volatility $\sigma$ the stock price increase, for example, fixing the strike $K=80$ the BS-solution increase from 17.9124 for $\sigma=0.5$ to 29.1376 for $\sigma=1.0$. On the other hand, the PUFEM and FEM results are closely identical.

For a better insight, we compare in table 3 the relative $L^{2}$-norm error obtained using both FE and PUFE methods. As it can be seen from the results, the obtained $L^{2}$-error using both methods are relatively small. However, the errors obtained using the PUFE method are smaller than the errors obtained using the FE method, for instance, for $K=120$ and $\sigma=1.0, \epsilon=$ 0.05064 using the FE method while $\epsilon=0.04567$ using the PUFE method. On the other hand, to achieve an error $\epsilon=0.03$ for $K=80$ and $\sigma=1.0,1000$ degrees of freedom are needed with the FEM whereas with the PUFEM this is reduced to 200.

The clear indication from this table is that, for different values of $K$ and $\sigma$, the computed errors using the PUFE method is smaller than the computed errors using the FE method. It is also evident that for a fixed strike $K$ more accurate results can be achieved by increasing the value of volatility $\sigma$, for example, for $K$ fixed to 80 the error decrease from $\epsilon=0.10356$ for $\sigma=0.5$ to $\epsilon=0.03512$ for $\sigma=1.0$. However, for a fixed volatility $\sigma$ the computed errors increase slightly by increasing the strike $K$. For example, for $\sigma=0.8$ and $K$ increase from 80 to 120 the computed errors increase from $\epsilon=0.04897$ to $\epsilon=0.08055$, the same conclusion can also be drawn by fixing $\sigma$ to 0.5 and 1 .

Figure 1 shows the solution surface of the American Put using both FE and PUFE methods, also five enrichment functions are used for the PUFE method. The considered meshes for the FE and PUFE methods are similar to the meshes considered previously where a finer mesh is used to compute the FEM solution with 1000 degrees of freedom, the obtained solution is considered as a reference solution. The PUFEM solution is obtained on a coarser mesh with 200 degress of freedom. Despite the much coarser mesh with the PUFE method, the plot show similar distributions patterns obtained using both methods. The obtained results show that the PUFE method is capable of recovering the solution variation on a coarser mesh using few enrichment functions.

To further compare the results obtained using the PUFE method to those obtained using the FE method, we plot in Figure 2 the variation of the stock price using the analytical solution, the FEM solution and the PUFEM solution. This figure shows a close match between the PUFE solution with the reference solution obtained using the FE method on a fine mesh. Yet, the PUFE is still better and more accurate than FE method if we compare it to the Black-Scholes analytic solution.

\section{Conclusions}

In this paper we have proposed a robust combination of the finite element and partition of unity methods to obtain effective numerical solutions of the linear and nonlinear American put option models represented by the Black-Scholes equation. The implementation of such a method by linearly implicit way is found efficient and stable when computing American pricing options. The convergence of the solution has been measured by some error norms and it is confirmed that the present method is asymptotically convergent. Furthermore, the produced 

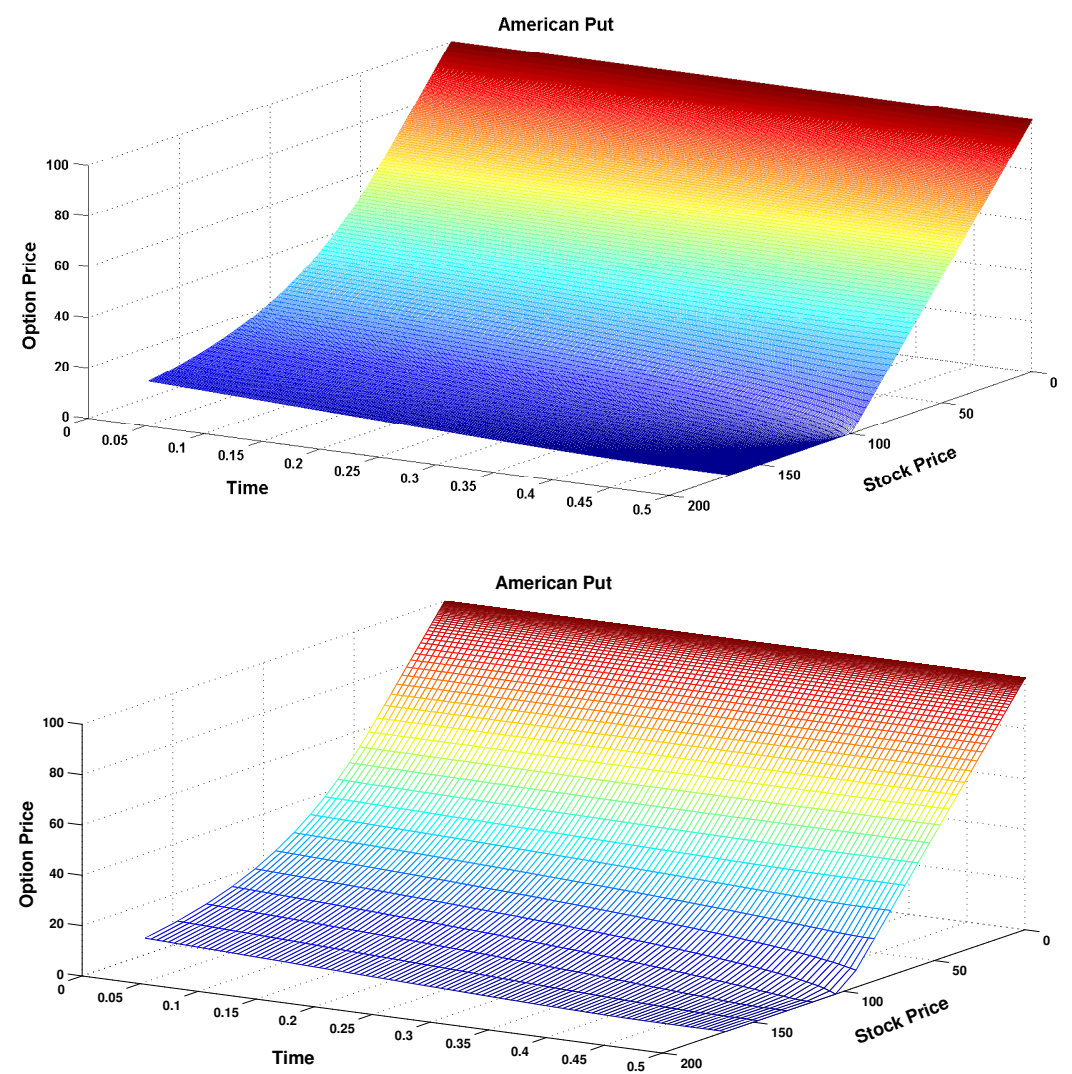

FIGURE 1. Solution surface for American Put obtained using the FE method (top) and the PUFE method (bottom) for the Black-Scholes problem.

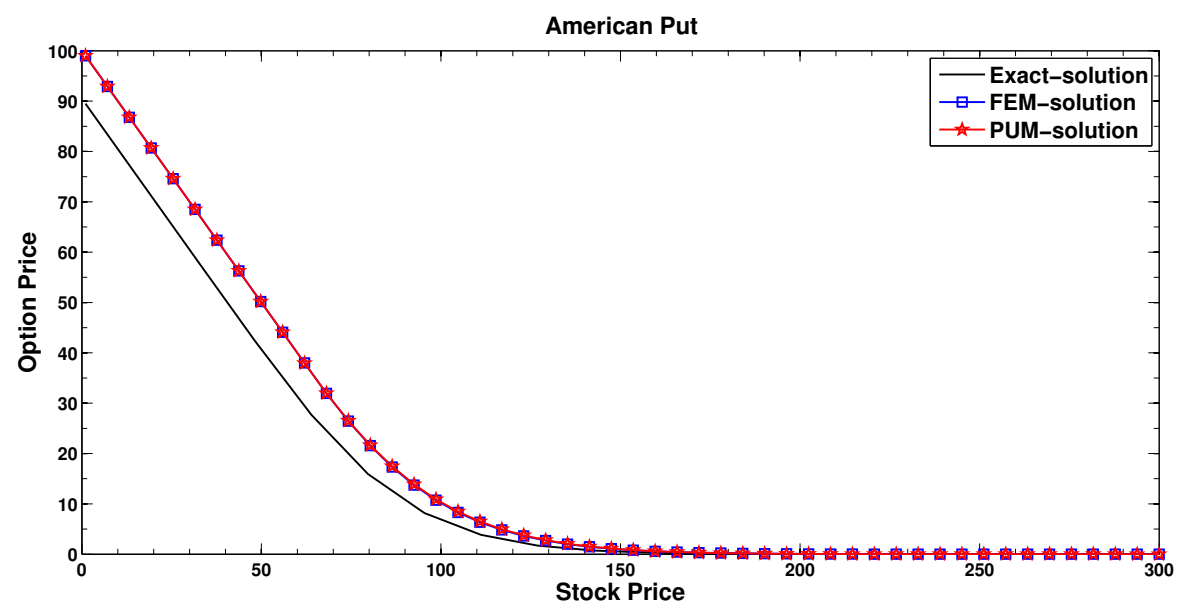

FigURE 2. Comparison between the Black-Scholes solution, the FEM solution and the PUFEM solution for American Put. 
results are in good agreement with the literature and the exact solution. Therefore, the proposed approach provides a better perspective to describe behavior of the option pricing model represented by the Black-Scholes equation and can be preferred due to reliability and accuracy with minimal computational effort. We believe that the numerical techniques presented in this paper make it promising to be extended in two-dimensions and also to be used in other similar fractional models for pricing different options. Further research is underway for tackling more complex and larger real option pricing problems using the PUFEM.

\section{References}

[1] Y. Achdou and O. Pironneau, Computational methods for option pricing, Society for Industrial and Applied Mathematics, (2005).

[2] Z. Al-Zhour, M. Barfeie, F. Soleymani, and E. Tohidi, A computational method to price with transaction costs under the nonlinear Black-Scholes model, CMES-Comp Model. Eng. , 124 (2020), 61-78.

[3] A. Andalaft-Chacur, M. M. Ali and J. G. Salazar, Real options pricing by the finite element method, Comput. Math. Appl. , 61 (2011), pp. 2863-2873.

[4] F. Black and M. Scholes, The pricing of options and corporate liabilities, J. Political Econ. , 81 (1973), 637-654.

[5] A. Chakraborty and B.V. R. Kumar, Finite element method for drifted space fractional tempered diffusion equation, J. Appl. Math. Comput. , 61 (2019), 117-135.

[6] M. M. Chawla and D. J. Evans, Numerical volatility in option valuation from black-scholes equation by finite differences, Int. J. Comput. Math. , 81 (2004), 1039-1041.

[7] C. De Schryver, FPGA Based Accelerators for Financial Applications, Springer International Publishing , (2015).

[8] A. EL Kahoui, M. Malek, N. Izem, M.S. Mohamed, and M. Seaid, Partition of Unity Finite Element Analysis of Nonlinear Transient Diffusion Problems using p-Version Refinement, CMES-Comp Model. Eng. , 124 (2020), 61-78.

[9] C. Feng, J. TAN, Z. JIANG, AND S. Chen, A generalized European option pricing model with risk management, Physica A. , 545 (2020), 123797.

[10] J. Liu, Two fast finite difference schemes for elliptic Dirichlet boundary control problems, J. Appl. Math. Comput. , 61 (2019), 481-503.

[11] F. Liu, I. Turner and V. Anh, An unstructured mesh finite volume method for modelling saltwater intrusion into coastal aquifers, J. Comput. \& Appl. Math., 9 (2002), 391-407.

[12] J. Lyu, E. Park, S. Kim, W. Lee, C. Lee, S. Yoon, J. Park, and J. Kim, Optimal non-uniform finite difference grids for the Black-Scholes equations, Math. Comput. Simul., 182 (2021), 690-704.

[13] M. Malek, N. Izem, M. Seaid, M.S. Mohamed, and M. Wakrim, A partition of unity finite element method for nonlinear transient diffusion problems in heterogeneous materials, Comp. Appl. Math. , 2 (2019), 31-38.

[14] J. M. Melenk and I. Babuška, The partition of unity finite element method: Basic theory and applications, Comput. Methods Appl. Mech. Engrg, 139 (1996), 289-314.

[15] M. Malek, N. Izem, M.S. Mohamed, and M. Seaid, O. Laghrouche, A partition of unity finite element method for three-dimensional transient diffusion problems with sharp gradients, J. Comput. Phys. , 396 (2019), 702-717.

[16] M. Malek, N. Izem, M.S. Mohamed, and M. Seaid, A three-dimensional enriched finite element method for nonlinear transient heat transfer in functionally graded materials, Int. J. Heat Mass Transf. , 155 (2020), 119804.

[17] F. Mercurio and Ton .C F. Vorst, Option pricing with hedging at fixed trading dates, Appl. Math. Finance , (2006), 135-158.

[18] M. S. Mohamed, M. Seaid, J. Trevelyan, and O. Laghrouche, A partition of unity fem for time-dependent diffusion problems using multiple enrichment functions, Int. J. Numer. Meth. Engng. , 93 (2013), 245-265.

[19] M. S. Mohamed, M. Seaid, J. Trevelyan, and O. Laghrouche, An enriched finite element model with qrefinement for radiative boundary layers in glass cooling, J. Comp. Phys. , 258 (2014), 718-737. 
[20] O. Turcoane, Option Price Estimations and Speculative Trading in Knowledge Society, Procedia Economics and Finance , 3(2012): 432-437.

[21] S. Wang, S. Zhang and Z. Fang, A superconvergent fitted finite volume method for black-scholes equations governing european and american option valuation, Numer. Methods Partial Differ. Equ. , 31(2015): 1190-1208.

[22] P. Wilmott, S. Howson, S. Howison, et al., The mathematics of financial derivatives: a student introduction, Cambridge university press, (1995). 